Memoirs of The College of Science, University of Kyoto, Series A

Vol. XXIX, Mathematics No. 1, 1955.

\title{
Kelvin principle and some inequalities in the theory of functions II
}

By

\author{
Tadao KUBo
}

(Recieved May, 1954)

In the previous paper ${ }^{3}$ the author established, by means of the Kelvin minimum energy principle, several inequalities which may be reduced to the statements regarding the properties of harmonic functions with a vanishing normal derivative on some of boundary components of a given domain.

It is the object of this paper to deduce further inequalities of the same kind and supplement the previous one. For the sake of simplicity we shall use the same notations with that of the previous paper.

1. Generalization of Theorem I. In this section we shall generalize Theorem I to the case of a domain $D$ which contains a number of mutually disjoint subdomains $D_{1}, D_{2}, \cdots, D_{n}$. We now have to introduce a number of singularity functions $S_{\nu}(z) \quad(\nu=1$, $\cdots, m$ ), which are harmonic and single-valued in the closure of $D$ $-D_{\nu}$. For the shorter formulation we restrict ourselves to the case of schlicht domains bounded by a finite number of analytic curves.

Thus we obtain the following

THEOREM III. Let $D$ be a schlicht domain and let $D_{1}, \cdots, D_{m}$ be mutually disjoint subdomains of $D$. Let $S_{\nu}(z) \quad(\nu=1, \cdots, m)$ be the singularity functions defined above and let the functions $p_{\nu}(z)$ $(\nu=1, \cdots, m)$ be such that $\partial p_{v} / \partial n=0$ on the boundary $C_{\nu}$ of $D_{v}$, and that $p_{\nu}(z)+S_{\nu}(z)$ is harmonic in $D_{\nu}$. If $P(z)$ is a function which has a vanishing normal derivative on the boundary $C$ of $D$ and for which $P(z)+\sum_{\nu=1}^{m} S_{\nu}(z)$ is harmonic in $D$, then

$$
\sum_{\nu=1}^{m} \int_{C_{\nu}} p_{\nu} \frac{\partial S}{\partial n} d s \geqq \int_{C} P \frac{\partial S}{\partial n} d s
$$


where $S(z)=\sum_{\nu=1}^{m} S_{\nu}(2)$ and the differentiation is performed with respect to the outer normal.

Proof. We define a function $u(z)$ as follows:

$$
\begin{array}{ll}
u(z)=p_{\nu}(z)+S(z) & z \in D_{\nu}(\nu=1, \cdots, m) \\
u(z)=S(z) & z \in D-\sum_{\nu=1}^{m} D_{\nu} .
\end{array}
$$

Although the function $u(z)$ is, in general, discontinuous on $C_{v}$, it has, by the assumption on $p_{v}(z)$, a common value of outward normal derivative along $C_{\nu}$ from both sides of it. Further consider the function

$$
v(z)=P(z)+S(z) \quad z \in D
$$

Then there holds

$$
\frac{\partial u}{\partial n}=\frac{\partial v}{\partial n} \text { on } C .
$$
Therefore the vector $\boldsymbol{q}=\left[\frac{\partial u}{\partial x}, \frac{\partial u}{\partial y}\right]$ satisfies the following condi-
tions:

(i) $\operatorname{div} \boldsymbol{q}=0$ everywhere in $D$ except along $C_{\nu}$,

(ii) $\boldsymbol{\Psi} \boldsymbol{n}=\frac{\partial u}{\partial n}=\frac{\partial v}{\partial n} \quad$ on $C, \boldsymbol{n}$ being unit normal vector.

Accordingly, by the Kelvin principle and Remark of Sec. 2 of the previous paper, there holds an inequality

$$
(v, v)_{\nu} \leqq(u, u)_{\nu}=\sum_{\nu=1}^{m}(u, u)_{D_{\nu}}+(u, u)_{D-\Sigma D_{\nu}},
$$

where $(h, k)_{D}$ denotes a Dirichlet product as follows:

$$
(h, k)_{D}=\iint_{D}\left(\frac{\partial h}{\partial x} \frac{\partial k}{\partial x}+\frac{\partial h}{\partial y} \frac{\partial k}{\partial y}\right) d x d y .
$$

Applying Green's formula to (4) and from (2) and (3),

$$
\text { the left-hand side of } \begin{aligned}
(4) & =\int_{C}(P+S) \cdot \frac{\partial(P+S)}{\partial n} d s \\
& =\int_{C} P \frac{\partial S}{\partial n} d s+\int_{C} S \frac{\partial S}{\partial n} d s,
\end{aligned}
$$

and

the right-hand side of (4) 
Kelvin principle and some inequalities in the theory of functions II 19

$$
\begin{aligned}
& =\sum_{\nu=1}^{m} \int_{C_{\nu}}\left(p_{\nu}+S\right) \frac{\partial\left(p_{\nu}+S\right)}{\partial n} d s+\int_{c} S \frac{\partial S}{\partial n} d s-\sum_{\nu=1}^{m} \int_{C_{\nu}} S \frac{\partial S}{\partial n} d s \\
& =\sum_{j=1}^{m} \int_{C_{\nu}} p_{\nu} \frac{\partial S}{\partial n} d s+\int_{c} S \frac{\partial S}{\partial n} d s .
\end{aligned}
$$

From (4), (5) and (6) we obtain the required inequality (1).

Q.E.D.

2. Neumann functions of mutually disjoint domains. As an application of Theorem III we deduce a result concerning Neumann functions of mutually disjoint subdomains $D_{j}(j=1, \cdots, m)$ of the domain $D$.

Take arbitrary two points $\zeta_{j}, \eta_{j}$ in $D_{j}(j=1, \cdots, m)$ and consider the functions ${ }^{1)}$

$$
\begin{aligned}
N\left(z ; \zeta_{j}, \eta_{j}\right) & =N\left(z, \zeta_{j}\right)-N\left(z, \eta_{j}\right) \\
& =\log \left|\frac{z-\eta_{j}}{z-\zeta_{j}}\right|+n\left(z ; \zeta_{j}, \eta_{j}\right) \quad(j=1, \cdots, m)
\end{aligned}
$$

and

$$
\begin{aligned}
N_{j}\left(z ; \zeta_{j}, \eta_{j}\right) & =N_{j}\left(z, \zeta_{j}\right)-N_{j}\left(z, \eta_{j}\right) \\
& =\log \left|\frac{z-\eta_{j}}{z-\zeta_{j}}\right|+n_{j}\left(z ; \zeta_{j}, \eta_{j}\right) \quad(j=1, \cdots, m)
\end{aligned}
$$

where $N(z, x)$ and $N_{j}(z, x) \quad(j=1, \cdots, m)$ are Neumann functions of $D$ and $D_{j}$, with a logarithmic singularity at $z=x$, respectively. A'nd further, putting

$$
\begin{aligned}
& S(z)=-\sum_{j=1}^{m} \alpha_{j} \log \left|\frac{z-\eta_{j}}{z-\zeta_{j}}\right|, \\
& p_{j}(z)=\alpha_{j} N_{j}\left(z ; \zeta_{j}, \eta_{j}\right), \quad(j=1, \cdots, m) \\
& P(z)=\sum_{j=1}^{m} \alpha_{j} N\left(z ; \zeta_{j} ; \eta_{j}\right),
\end{aligned}
$$

$\alpha_{j}$ being arbitrary real constants, we can use Theorem III. Then we obtain

$$
\begin{aligned}
\sum_{j=1}^{m} \int_{C_{j}} p_{j} \frac{\partial S}{\partial n} d s= & -\sum_{j=1}^{m}\left\{\int_{C_{j}} \alpha_{j} N_{j}\left(z ; \zeta_{j}, \eta_{j}\right) \frac{\partial}{\partial n}\left(\sum_{i=1}^{m} \alpha_{i} \log \left|\frac{z-\eta_{i}}{z-\zeta_{i}}\right|\right) d s\right\} \\
= & -\sum_{j=1}^{m} \alpha_{j}^{2} \int_{C_{j}} N_{j}\left(z ; \zeta_{j}, \eta_{j}\right) \frac{\partial}{\partial n} \log \left|\frac{z-\eta_{j}}{z-\zeta_{j}}\right| d s \\
& -\sum_{i \neq j}^{m} \alpha_{i} \alpha_{j} \int_{C_{j}} N_{j}\left(z ; \zeta_{j}, \eta_{j}\right) \frac{\partial}{\partial n} \log \left|\frac{z-\eta_{i}}{z-\zeta_{i}}\right| d s
\end{aligned}
$$




$$
\begin{aligned}
= & \sum_{j=1}^{m} \alpha_{j}^{2} \int_{C_{j}} N_{j}\left(2 ; \zeta_{j}, \eta_{j}\right) \frac{\partial}{\partial n} n_{i}\left(2 ; \zeta_{j}, \eta_{j}\right) d s \\
& -\sum_{i \neq j}^{m} \alpha_{i} \alpha_{j} \int_{C_{j}} N_{j}\left(2 ; \zeta_{j}, n_{j}\right) \frac{\partial}{\partial n} \log \left|\frac{z-\eta_{i}}{z-\zeta_{i}}\right| d s .
\end{aligned}
$$

For, since $\frac{\partial N_{j}}{\partial n}=0$ on $C_{j},-\frac{\partial}{\partial n} \log \left|\frac{z-\eta_{j}}{z-\tau_{j}}\right|=\frac{\partial}{\partial n} n_{j}\left(z ; \zeta_{j}, \eta_{j}\right)$ on $C_{j}$. Here we apply the well-known formula ${ }^{4)}$ of Neumann function such that for any harmonic function $U(z)$ it holds

$$
\frac{1}{2 \pi} \int_{C_{j}} N_{j}\left(z ; \zeta_{j}, \eta_{j}\right) \frac{\partial U(z)}{\partial n} d s=U\left(\zeta_{j}\right)-U\left(\eta_{j}\right), \quad(j=1, \cdots, m) .
$$

Then we have

$$
\begin{aligned}
\sum_{j=1}^{m} \int_{c_{j}} p_{j} \frac{\partial S}{\partial n} d s=2 \pi \sum_{j=1}^{m} \alpha_{j}^{2}\left[n_{j}\left(\zeta_{j} ; \zeta_{j}, \eta_{j}\right)-n_{j}\left(\eta_{j} ; \zeta_{j}, \eta_{j}\right)\right] \\
+4 \pi \sum_{i<j}^{m} \alpha_{i} \alpha_{j} \log \left|\frac{\zeta_{j}-\zeta_{i}}{\zeta_{j}-\eta_{i}} \cdot \frac{\eta_{j}-\eta_{i}}{\eta_{j}-\zeta_{i}}\right| .
\end{aligned}
$$

Similarly we obtain

$$
\int_{c} P \frac{\partial S}{\partial n} d s=2 \pi \sum_{i, j=1}^{m} \alpha_{i} \alpha_{j}\left[n\left(\zeta_{j} ; \zeta_{i}, \eta_{i}\right)-n\left(\eta_{j} ; \zeta_{i}, \eta_{i}\right)\right]
$$

Thus we obtain the following

Corollary 8. Let $D_{i}(j=1, \cdots, m)$ be mutually disjoint subdomains of the domain $D$ and let $N(z, \zeta)$ and $N_{i}(z, \zeta) \quad(j=1, \cdots$, $m$ ) be Neumann functions of the domain $D$ and $D_{j}$, respectively. Then there holds the following inequality

$$
\begin{aligned}
& \sum_{j=1}^{m} \alpha_{j}^{2}\left[n_{j}\left(\zeta_{j} ; \zeta_{j}, \eta_{j}\right)-n_{j}\left(\eta_{j} ; \zeta_{j}, \eta_{j}\right)\right]+2 \sum_{i<i}^{m} \alpha_{i} \alpha_{j} \log \left|\frac{\zeta_{i}-\zeta_{j}}{\eta_{i}-\zeta_{j}} \cdot \frac{\eta_{i}-\eta_{j}}{\zeta_{i}-\eta_{j}}\right| \\
& \quad \geqq \sum_{i j=1}^{m} \alpha_{i} \alpha_{j}\left[n\left(\zeta_{j} ; \zeta_{i}, \eta_{i}\right)-n\left(\eta_{j} ; \zeta_{i}, \eta_{j}\right)\right],
\end{aligned}
$$

where $\alpha_{j}$ are arbirary real constants.

3. Variation of outer boundary component. Let $D$ and $D_{1}$ be two finite multiply-connected domains such that $D_{1}$ contains $D$ and is obtained only by a variation of outer boundary component of $D$, remaining fixed all the inner boundary components of $D$. For the definiteness we denote the total boundary curves of two domains $D$ and $D_{1}$ by $\gamma+\sum_{\nu=1}^{n} C_{\nu}$, and $\gamma_{1}+\sum_{\nu=1}^{n} C_{\nu}, \gamma$ and $\gamma_{1}$ being outer boundary components of $\stackrel{\nu=1}{D}$ and $D_{1}$, respectively, and $C_{\nu}(\nu=1, \cdots, n)$ being 
Kelvin principle and some inequalities in the theory of functions II 21 common inner boundary components. For the sake of simplicity we assume that $D_{1}-\bar{D}$ be a ring domain. Then we obtain the following

THEOREM IV: Let $D$ and $D_{1}$ be two domains as described above and $S(z)$ have the same meaning as in Theorem I. Let $p(z)$ denote the function satisfying the following conditions: (i) $p(z)=0$ on $\gamma$, (ii) $\partial p / \partial n=0$ on each inner boundary component $C_{\nu}$, (iii) $p+S$ is harmonic in $D$, (iv) $\int_{\beta} \partial(p+S) / \partial n d s=0$ for any closed path $\beta$ topologically equivalent to $r$ in $D$. If $p_{1}(z)$ is the corresponding function associated with $D_{1}$, and moreover $\omega(z)$ is the harmonic measure which has the boundary values 1 and 0 on $r$ and $\gamma_{1}$, respectively, then there holds the following inequality

$$
\begin{aligned}
& \int_{\tau} S \frac{\partial p}{\partial n} d s-\sum_{\nu} \int_{C_{\nu}} p \frac{\partial S}{\partial n} d s \\
& \quad \geqq \int_{T_{1}} S \frac{\partial p_{1}}{\partial n} d s-\sum_{\nu} \int_{C_{\nu}} p_{1} \frac{\partial S}{\partial n} d s+\frac{(S, \omega)^{2} D_{1}-\bar{D}}{(\omega, \omega)_{D_{1}-\bar{D}}} .
\end{aligned}
$$

Proof. At first we formulate various relations between three functions $p_{1}(z), S(z)$ and $\omega(z)$ harmonic in $D_{1}-\bar{D}$. Applying Green's identity in the domain $D_{1}-\bar{D}$, we obtain the following relations

$$
\int_{r_{1}} S \frac{\partial \omega}{\partial n} d s=\int_{r_{1}}\left(S \frac{\partial \omega}{\partial n}-\omega \frac{\partial S}{\partial n}\right) d s=\int_{r}\left(S \frac{\partial \omega}{\partial n}-\frac{\partial S}{\partial n}\right) d s .
$$

And further

$$
\int_{r}\left(S \frac{\partial p_{1}}{\partial n}-p_{1} \frac{\partial S}{\partial n}\right) d s=\int_{r_{1}}\left(S \frac{\partial p_{1}}{\partial n}-p_{1} \frac{\partial S}{\partial n}\right) d s=\int_{r_{1}} S \frac{\partial p_{1}}{\partial n} d s .
$$

From the relation

$$
\begin{aligned}
& \int_{r}\left(p_{1} \frac{\partial \omega}{\partial n}-\omega \frac{\partial p_{1}}{\partial n}\right) d s=\int_{r_{1}}\left(p_{1} \frac{\partial \omega}{\partial n}-\omega \frac{\partial p_{1}}{\partial n}\right) d s=0, \\
& \int_{\tau} p_{1} \frac{\partial \omega}{\partial n} d s=\int_{r} \omega \frac{\partial p_{1}}{\partial n} d s=\int_{r} \frac{\partial p_{1}}{\partial n} d s=-\int_{\Upsilon} \frac{\partial S}{\partial n} d s,
\end{aligned}
$$

because of the condition (iv) for $p_{1}(z)$.

After above preparatory consideration we define two functions $u(z)$ and $v(z)$ as follows:

$$
\begin{array}{ll}
u(z)=p(z)+S(z)+\alpha & z \in D, \\
u(z)=S(z)+\alpha \omega(z) & z \in D_{1}-\bar{D},
\end{array}
$$


$\alpha$, being arbitrary real constant, and

$$
v(z)=p_{1}(z)+S(z) \quad z \in D_{1} .
$$

It is obvious that the function $u(z)$ is not only continuous in $D$, but also continuous in $D_{1}-\bar{D}$ beyond $\gamma, u=v$ on $\gamma_{1}$ and $\partial u / \partial n=$ $\partial v / \partial n$ on $C_{\nu}$. From the identity

$$
(v, v)_{p_{1}}=(v-u, v-u)_{D_{1}}+2(v-u, u)_{D_{1}}+(u, u)_{D_{1}}
$$

we obtain an inequality

$$
(u, u)_{p_{2}}-2(u-v, u)_{D_{1}}-(v, v)_{D_{1}} \leqq 0
$$

Remarking that $u(z)$ is stepwisely differentiable in $D_{1}$, and from the definition of $u(z)$, we get the following relations.

$$
\begin{aligned}
& (u, u)_{D_{1}}=(u, u)_{\nu}+(u, u)_{D_{1}-\bar{D}} \\
& =(p+S, p+S)_{D}+\left(S+\alpha \omega, S+\alpha(v)_{D_{1}-\vec{D}}\right. \\
& =\sum_{\nu} \int_{C_{\nu}}(p+S) \frac{\partial(p+S)}{\partial n} d s+\int_{r}(p+S) \frac{\partial(p+S)}{\partial n} d s \\
& -\int_{\gamma}\left(S+\alpha(v) \frac{\partial(S+\alpha(v)}{\partial n} d s+\int_{\gamma_{1}}\left(S+\alpha(v) \frac{\partial(S+\alpha(t))}{\partial n} d s\right.\right. \\
& =\sum_{\nu} \int_{C_{\nu}}(p+S) \frac{\partial S}{\partial n} d s+\int_{r} S \frac{\partial p}{\partial n} d s-\alpha \int_{r} \frac{\partial S}{\partial n} d s \\
& -\alpha \int_{\curlyvee} S \frac{\partial \omega}{\partial n} d s-\alpha^{2} \int_{\curlyvee} \frac{\partial \omega}{\partial n} d s+\int_{\gamma_{1}} S \frac{\partial S}{\partial n} d s+\alpha \int_{\Upsilon_{1}} S \frac{\partial \omega}{\partial n} d s,
\end{aligned}
$$

$(v, v)_{p_{1}}=\left(p_{1}+S, p_{1}+S\right)_{p_{1}}$

$$
\begin{aligned}
& =\sum_{\nu} \int_{C_{\nu}}\left(p_{1}+S\right) \frac{\partial\left(p_{1}+S\right)}{\partial n} d s+\int_{r_{1}}\left(p_{1}+S\right) \frac{\partial\left(p_{1}+S\right)}{\partial n} d s \\
& =\sum_{\nu} \int_{C_{\nu}}\left(p_{1}+S\right) \frac{\partial S}{\partial n} d s+\int_{r_{1}} S \frac{\partial p_{1}}{\partial n} d s+\int_{r_{1}} S \frac{\partial S}{\partial n} d s,
\end{aligned}
$$

and

$$
\begin{aligned}
(u, u-v)_{D_{1}}= & (u, u-v)_{D}+(u, u-v)_{D_{1}-\bar{D}} \\
= & \sum_{\nu} \int_{C_{\nu}} u \frac{\partial(u-v)}{\partial n} d s+\int_{r} u \frac{\partial(u-v)}{\partial n} d s \\
& -\int_{\gamma}(u-v) \frac{\partial u}{\partial n} d s+\int_{\gamma_{1}}(u-v) \frac{\partial u}{\partial n} d s
\end{aligned}
$$

(the 1 st and 4 -th integrals vanish by the above remark) 
Kelvin principle and some inequalities in the theory of functions II 23

$$
\begin{gathered}
=\int_{r}(p+S+\alpha) \frac{\partial\left(p-p_{1}\right)}{\partial n} d s-\int_{r}\left(\alpha(1)-p_{1}\right)\left(\frac{\partial S}{\partial n}+\alpha \frac{\partial(1)}{\partial n}\right) d s \\
=\int_{r} S \frac{\partial p}{\partial n} d s-\int_{r} S \frac{\partial p_{1}}{\partial n} d s-\alpha \int_{r} \frac{\partial S}{\partial n} d s-\alpha^{2} \int_{r} \frac{\partial \omega}{\partial n} d s \\
+\int_{r} p_{1} \frac{\partial S}{\partial n} d s+\alpha \int_{r} p_{1} \frac{\partial \omega}{\partial n} d s
\end{gathered}
$$

because of $\int_{r} \frac{\partial\left(p_{1}-p\right)}{\partial n} d s=0$ from the conditions (iv) for $p(z)$ and $p_{1}(z)$.

From (16) and (17)

$$
\begin{aligned}
& (u, u)_{\nu_{1}}-(v, v)_{m_{1}}=\sum_{v} \int_{C_{v}} p_{\nu} \frac{\partial S}{\partial n} d s+\int_{\tau} S \frac{\partial p}{\partial n} d s-\sum_{v} \int_{C_{\nu}} p_{v} \frac{\partial S}{\partial n} d s \\
& -\int_{\tau_{1}} S \frac{\partial p_{1}}{\partial n} d s-\alpha \int_{r} \frac{\partial S}{\partial n} d s-\alpha \int_{r} S \frac{\partial \omega}{\partial n} d s+\alpha \int_{\gamma_{1}} S \frac{\partial \omega}{\partial n} d s \\
& \text { (19) } \quad-\alpha^{2} \int_{r} \frac{\partial \omega}{\partial n} d s \\
& =\sum_{\nu} \int_{C_{\nu}} p \frac{\partial S}{\partial n} d s+\int_{r} S \frac{\partial p}{\partial n} d s-\sum_{\nu} \int_{C_{\nu}} p_{1} \frac{\partial S}{\partial n} d s-\int_{r_{1}} S \frac{\partial p_{1}}{\partial n} d s \\
& -2 \alpha \int_{\curlyvee} \frac{\partial S}{\partial n} d s-\alpha^{2} \int_{r} \frac{\partial \omega}{\partial n} d s,
\end{aligned}
$$

by applying (12) to the 7 -th term.

And further, from (18),

$$
\begin{aligned}
-2(u, & u-v)_{D_{1}}=-2 \int_{r} S \frac{\partial p}{\partial n} d s+2\left(\int_{r} S \frac{\partial p_{1}}{\partial n} d s-\int_{r} p_{1} \frac{\partial S}{\partial n} d s\right) \\
& +2 \alpha \int_{r} \frac{\partial S}{\partial n} d s-2 \alpha \int_{r} p_{1} \frac{\partial(\omega)}{\partial n} d s+2 \alpha^{2} \int_{r} \frac{\partial(1)}{\partial n} d s \\
= & -2 \int_{r} S \frac{\partial p}{\partial n} d s+2 \int_{r_{1}} S \frac{\partial p_{1}}{\partial n} d s+4 \alpha \int_{r} \frac{\partial S}{\partial n} d s+2 \alpha^{2} \int_{r} \frac{\partial \omega}{\partial n} d s,
\end{aligned}
$$

by applying (13) and (14) to the 2nd and 4-th term, respectively. Inserting (19) and (20) into (15), and remarking that $(\omega, \omega)_{D_{1}-\bar{J}}$

$$
\begin{aligned}
=- & \int_{r} \frac{\partial \omega}{\partial n} d s, \text { and }(S, \omega)_{D_{1}-\bar{D}}=-\int_{r} \frac{\partial S}{\partial n} d s, \\
& \sum_{\nu} \int_{C_{\nu}} p \frac{\partial S}{\partial n} d s-\int_{r} S \frac{\partial p}{\partial n} d s
\end{aligned}
$$




$$
\leqq \sum \int_{\nu} p_{C_{\nu}} \frac{\partial S}{\partial n} d s-\int_{\tau_{1}} S \frac{\partial p_{1}}{\partial n} d s+2 \alpha(S, \omega)_{p_{1}-\bar{D}}+\alpha^{2}(\omega, \omega)_{p_{1}-\bar{D}}
$$

The best inequality of this type is obtained for

$$
\alpha=-\frac{\left(S,(\omega)_{D_{1}-\bar{p}}\right.}{(\omega, \omega)_{D_{1}-\bar{D}}}
$$

If we choose this value of $\alpha$ in the above inequality, we obtain the required result (11).

Remark. Since the inequality (15) is deduced from the positive definite character of $(u-v, u-v)_{D_{1}}$, there holds the equality in (11) if and only if $p_{1}(z)=p(z)+\alpha$ in $D$ and $p_{1}(z)=\alpha \omega(z)$ in $D_{1}-\bar{D}$, in other words, $p_{1}(z)=$ const. on the outer boundary component $r$ of the domain $D$.

4. Some applications of Theorem IV. In this section we shall apply the above theorem to the bounded radial slit mapping functions of $D$ and $\dot{D}_{1}$ as mentioned in Sec. 6 of the previous paper. The existence of such a mapping function was verified by Koebe ${ }^{2)}$ by potential-theoretic method.

We denote by $w=F(z, \zeta)(F(\zeta, \zeta)=0, \zeta \in D)$ the analytic function which maps $D$ onto the unit circle slit along radial segments directed towards the origin such that the outer boundary component $\gamma$ of $D$ be transformed into the unit circumference. Moreover we denote by $F_{1}(2, \zeta)\left(F_{1}(\zeta, \zeta)=0, \zeta \in D\right)$ the mapping function of the same type associated with $D_{1}$. Then the harmonic functions

$$
\begin{gathered}
p(z)=\log |F(z, \zeta)|, S(z)=-\log |z-\zeta|, \\
\text { and } \quad p_{1}(z)=\log \left|F_{1}(z, \zeta)\right|
\end{gathered}
$$

satisfy all the conditions of Theorem IV. As obtained in Sec. 6 of the previous paper

$$
\int_{r} S \frac{\partial p}{\partial n} d s-\sum_{\nu} \int_{C_{\nu}} p \frac{\partial S}{\partial n} d s=2 \pi \log \left|F^{\prime}(\zeta, \zeta)\right|,
$$

and as is well-known,

$$
(\omega, \omega)_{D_{1}-\bar{D}}=\frac{2 \pi}{\log M},
$$

$M$ being the Riemann modulus of the ring domain $D_{1}-\bar{D}$. Further

$$
(S, \omega)_{D_{1}-\bar{D}}=-\int_{\mathrm{r}} \frac{\partial S}{\partial n} d s
$$


Kelvin principle and some inequalities in the theory of functions II 25

$$
\begin{aligned}
& =\int_{\tau} \frac{\partial}{\partial n} \log |z-\zeta| d s \\
& =\int_{\tau} \operatorname{darg}(z-\zeta)=2 \pi .
\end{aligned}
$$

By (21), (22), (23) and Theorem IV

$$
\log \left|F^{\prime}(\zeta, \zeta)\right| \geqq \log \left|F_{1}^{\prime}(\zeta, \zeta)\right|+\log M .
$$

Thus we have the following

Corollary 9. Let $D$ and $D_{1}\left(D \subset D_{1}\right)$ be two domains mentioned in the previous section. Further let $F(z, \zeta)$ and $F_{1}(z, \zeta)$ be the bounded radial slit mapping functions so normalized as described above of $D$ and $D_{1}$, respectively. Then there holds an inequality

$$
\left|F^{\prime}(\zeta, \zeta)\right| \geqq M\left|F_{1}^{\prime}(\zeta, \zeta)\right|,
$$

$M$ being the Riemann modulus of the ring domain $D_{1}-\bar{D}$.

It is obvious by Remark of the previous section that the equality holds in (24) if and only if the outer boundary component $\gamma$ of $D$ coincides with one of the level curves of the function $F_{1}(z, \zeta)$, i.e. $\left|F_{1}(z, \zeta)\right|=$ const. In fact, if $w=F_{1}(z, \zeta)$ maps $\gamma$ onto a concentric circle $|w|=Q \quad(<1)$, there holds

$$
F(z, \zeta)=\frac{e^{i \theta}}{Q} F_{1}(z, \zeta) \quad(\theta: \text { a real number })
$$

therefore

$$
\left|F^{\prime}(\zeta, \zeta)\right|=\frac{1}{Q}\left|F_{1}^{\prime}(\zeta, \zeta)\right| .
$$

Obviously $1 / Q$ equals to the Riemann modulus $M$ of $D_{1}-\bar{D}$.

Considering the limiting case of (24) when each inner boundary component $C_{\nu}$ converges to a points, we obtain the following corollary concerning the inner conformal radii ${ }^{\text {(i) }}$ of $D$ and $D_{1}$.

Corollary $10 .^{\mathrm{E})} \quad$ Let $D$ and $D_{1}$ be two simply-connected domains such that $D \subset D_{1}$ and let $r(\zeta)$ and $R(\zeta)$ denote the inner conformal radii of $D$ and $D_{1}$, respectively, with respect to a point $\zeta \in D$. If $M$ denotes the Riemann modulus of the ring domain $D_{1}-\bar{D}$, then

$$
M \leqq \frac{R(\zeta)}{r(\zeta)} .
$$

It will easily follow from (24) by noticing that in the case of simply-connected domain 


$$
\left|F^{\prime}(\zeta, \zeta)\right|=\frac{1}{r(\zeta)} \quad \text { and } \quad\left|F_{1}^{\prime}(\zeta, \zeta)\right|=\frac{1}{R(\zeta)}
$$

\section{Kyoto University}

\section{BIBLIOGRAPHY}

1. Bergman, $S$, The kernel function and conformal mapping, New York, Amer. Math. Soc. (1950).

2. Koebe, $\mathrm{P}$, Abhandlungen zur Theorie der konformen, Abbildung, IV. Abbildung mehrfach zusammenhängender schlichter Bereiche auf schlitzbereiche. Acta math. Vol. 41 (1918), pp. 305-344.

3. Kubo, T, Kelvin principle and some inequalities in the theory of functions I, Mem. Coll. Sci. Kyoto. Ser. A. Vol. 28 (1953), pp: 299-311

4. Nehari, Z, Conformal mapping, New York (1952). Especially see p. 25.

5. Nehari, $Z$, Some inequalities in the theory of functions, Trans. Amer. Math. Soc. Vol. 75 (1953) pp. 256-286.

6. Pólya, G and Szegö, G, Isoperimetric inequalities in mathematical physics, Princeton Univ. Press, (1951). 